\title{
RNA localization in neurite morphogenesis and synaptic regulation: current evidence and novel approaches
}

\author{
Martin Mikl · Georgia Vendra • Michael Doyle $\cdot$ \\ Michael A. Kiebler
}

Received: 18 December 2009 / Revised: 4 March 2010 / Accepted: 4 March 2010 / Published online: 17 March 2010

(C) The Author(s) 2010. This article is published with open access at Springerlink.com

\begin{abstract}
It is now generally accepted that RNA localization in the central nervous system conveys important roles both during development and in the adult brain. Of special interest is protein synthesis located at the synapse, as this potentially confers selective synaptic modification and has been implicated in the establishment of memories. However, the underlying molecular events are largely unknown. In this review, we will first discuss novel findings that highlight the role of RNA localization in neurons. We will focus on the role of RNA localization in neurotrophin signaling, axon outgrowth, dendrite and dendritic spine morphogenesis as well as in synaptic plasticity. Second, we will briefly present recent work on the role of microRNAs in translational control in dendrites and its implications for learning and memory. Finally, we discuss recent approaches to visualize RNAs in living cells and their employment for studying RNA trafficking in neurons.
\end{abstract}

Keywords Axonal and dendritic RNA localization · Translational control at the synapse $\cdot$ RNA-binding proteins (RBPs) · Dendritic spine morphogenesis · RNA imaging

$\begin{array}{ll}\text { Abbreviations } \\ \text { 3'-UTR } & 3^{\prime} \text {-Untranslated region } \\ \text { Arc or Arg3.1 } & \begin{array}{l}\text { Activity-regulated cytoskeleton-associated } \\ \text { protein }\end{array} \\ \text { BDNF } & \begin{array}{l}\text { Brain-derived neurotrophic factor } \\ \text { CaMKII } \alpha\end{array} \\ & \begin{array}{l}\alpha \text {-Subunit of the calcium/calmodulin- } \\ \text { dependent protein kinase II }\end{array}\end{array}$

M. Mikl · G. Vendra $\cdot$ M. Doyle $\cdot$ M. A. Kiebler $(\bowtie)$

Center for Brain Research, Medical University of Vienna,

Spitalgasse 4, 1090 Vienna, Austria

e-mail: michael.kiebler@meduniwien.ac.at

URL: http://www.meduniwien.ac.at/kieblerlab

$\begin{array}{ll}\text { CREB } & \text { cAMP-responsive element binding protein } \\ \text { CPEB } & \begin{array}{l}\text { Cytoplasmic polyadenylation element } \\ \text { binding protein }\end{array} \\ \text { FMRP } & \text { Fragile X mental retardation protein } \\ \text { GFP } & \text { Green fluorescent protein } \\ \text { LTD } & \text { Long-term depression } \\ \text { LTP } & \text { Long-term potentiation } \\ \text { mEPSC } & \text { Miniature excitatory postsynaptic current } \\ \text { MB } & \text { Molecular beacon } \\ \text { mTOR } & \text { Mammalian target of rapamycin } \\ \text { miRNA } & \text { Micro RNA } \\ \text { nos } & \text { Nanos RNA } \\ \text { NGF } & \text { Nerve growth factor } \\ \text { P-bodies } & \text { Processing bodies } \\ \text { RBP } & \text { RNA-binding protein } \\ \text { RISC } & \text { RNA-induced silencing complex } \\ \text { Stau2 } & \text { Double-stranded binding protein Staufen } 2\end{array}$

\section{Introduction}

RNA localization is a widespread mechanism that targets mRNAs to regions of the cell, where the proteins are required. Restricting proteins to spatially confined areas results in compartmentalization of the cell and can be achieved either by specific translocation of the protein or the accumulation of the mRNA at a specific area. In the latter case, protein expression can be controlled locally allowing the cell to quickly respond to environmental cues, including extracellular stimuli. This strategy is employed widely across species ranging from yeast to Drosophila and mammals and also within different cell types of a given organism, including neurons (St Johnston 2005).

As neurons are highly polarized cells they require sophisticated regulatory mechanisms to confer spatial 
resolution at every stage of development (Arimura and Kaibuchi 2007; Hengst and Jaffrey 2007; Barnes and Polleux 2009). Moreover, as these cells have extended processes, long physical distances exist between the soma and the distal axonal and dendritic compartments, further complicating fast responses to environmental changes or synaptic input. It is therefore favorable for certain responses to be independent from de novo RNA or protein synthesis in the cell body, and instead to rely on localized pools of RNAs that can be rapidly translated when required.

There have been numerous studies investigating the mechanism and functional components of RNA localization in neurons (Kiebler and DesGroseillers 2000; Sutton and Schuman 2006; Martin and Ephrussi 2009). Here, we update and extend on these findings specifically focusing on the physiological significance of RNA sorting for synaptic function, e.g., synaptic plasticity. We further discuss the molecular mechanism of translational control at the synapse and review recent evidence on the role of microRNAs (miRNAs) and their targets in translational silencing and their potential contribution to learning and memory. Finally, we describe the first attempts to visualize RNA transport in living neurons and unravel the molecular mechanism of dendritic RNA localization.

\section{The role of RNA localization in axon outgrowth}

Most work on RNA transport and local translation in neurons has been performed on dendritically localized RNAs, while only a limited number of localized transcripts have been identified in axons. For the most prominent axonally localized transcript, $\beta$-actin mRNA, the mechanisms regulating its translocation and translation are relatively well understood. In a recent study, convincing evidence was provided linking local translation of $\beta$-actin mRNA to steering of axonal growth cones (Leung et al. 2006). Here, the cDNA encoding the photoconvertible protein Kaede was fused to the $3^{\prime}$-UTR of the $\beta$-actin mRNA that contains the cis-acting elements regulating its localization and translation. As Kaede normally fluoresces green but shifts to red after UV irradiation (Ando et al. 2002), the authors were able to distinguish between protein present at a defined time point (i.e., the time of photoconversion), which will appear as red, and protein synthesized thereafter in green. They monitored the behavior of this reporter, which should resemble the characteristics of $\beta$-actin mRNA, in Xenopus retinal primordial cells and found it-like $\beta$-actin mRNAto be present in axonal growth cones. After photoconversion of existing Kaede protein, the neurotrophin Netrin-1 was applied to induce local translation of the reporter, observable by green fluorescence at the site of Netrin-1 action. This occurred ipsilaterally to the area of application and coincided with increased phosphorylation of the translation initiation factor eIF4E-BP. Stimulation of translation of local $\beta$-actin mRNA, however, happened only in attractive growth cone turning. When the repellents Sema3A and Slit2 were applied, no increase in $\beta$-actin synthesis was observed. This is in agreement with the view that repulsive turning represents local collapse of cell extensions mediated, e.g., by induced synthesis of actin depolymerizing factor/cofilin (general review on actin, see Pollard and Cooper 2009), rather than increased contralateral $\beta$-actin mRNA translation.

Similar to Holt et al., Yao et al. (2006) provided a link between growth cone turning and local $\beta$-actin synthesis in Xenopus neurons. They not only showed asymmetrical increase in $\beta$-actin upon local BDNF application close to one side of the growth cone, but also shed light on the mechanisms regulating this effect. On the one hand, they demonstrated its dependence on $\mathrm{Ca}^{2+}$ influx, which had already been linked to BDNF signaling (Song et al. 1997). On the other hand, they correlated increased $\beta$-actin mRNA translation with activation of Src by phosphorylation, which is a critical event in relieving ZBP1-mediated translational repression of $\beta$-actin mRNA (Hüttelmaier et al. 2005). Upon induction of repulsive turning, Src is deactivated at the site of application of the repellent, while the contralateral side is unaffected, resulting in an asymmetry in $\beta$-actin synthesis suggesting a Src-dependent effect to account for both attractive and repulsive turning. In this case, it is not cofilin-mediated actin depolymerization (general review on actin, see Pollard and Cooper 2009), but sustained repression of local actin translation that leads to the asymmetry observed.

The most prominent and best-studied examples of axon localized mRNAs have been observed during, and linked to, outgrowth and pathfinding of a developing axon. In addition, transcripts localizing to mature axons and the functional significance of this process have also been identified. A new study (Cox et al. 2008) reports an example of axonal RNA localization and subsequent local translation that not only leads to a spatially restricted response, but has an impact on the whole cell and is crucial for its survival. Jaffrey et al. show that the RNA encoding cAMP-responsive element binding protein (CREB) is found in axons of neurons from dorsal root ganglia, and is translated specifically in the axonal compartment upon local nerve growth factor (NGF) application. It is then retrogradely trafficked by active transport along the microtubule network. Under selective NGF application to axons, nuclear accumulation of phosphorylated CREB was shown to depend on this process. The same applies to the increase in CRE-dependent transcription and NGF-induced retrograde survival, which are triggered by axonally translated CREB as well. These results indicate the existence of a retrograde signaling 
pathway based on the presence of a transcript in the axon, allowing for specific regulation of transcription in the nucleus by signals encountered in distal processes.

In a more recent study, Jaffrey et al. (Hengst et al. 2009) took advantage of an elaborate experimental approach, when they cultured rat embryonic dorsal root ganglia in a compartmentalized microfluidic chamber (Taylor et al. 2005). This allows application of NGF exclusively to axons, which are found in a physically distinct part of the chamber. The authors demonstrated that Par3 mRNA is localized to developing axons, where NGF and Netrin-1 induce local translation. In neurons that lack axonal Par3 mRNA, stimulated axonal outgrowth is inhibited. In contrast, basal outgrowth appears not to be affected (Hengst et al. 2009). The PAR complex, consisting of PAR3, PAR6 and aPKC, specifies the axon during early development (Shi et al. 2003) and the authors propose in their new study a novel mechanism to regulate the complex by local translation of PAR3 mRNA in stimulated axons.

Similar events are thought to occur after nerve lesions. Retrograde signaling to the cell body triggered by axon injury has been shown to depend on local translation of importin $\beta 1$ (Hanz et al. 2003) and RanBP1 mRNA (Yudin et al. 2008). By binding the small GTPase Ran, RanBP1 enables the formation of an injury-signaling complex, consisting of-among others-importin- $\alpha$, locally synthesized importin $\beta 1$ and the microtubule minus-end directed molecular motor dynein. The importin heterodimer can then bind a protein containing a nuclear localization signal (NLS) with high efficiency and, by linking it to a cell body directed motor protein, promote its retrograde transport. Among these cargo proteins, the intermediate filament component Vimentin has been identified, which, like Importin- $\beta 1$ and RanBP1, is translated locally upon nerve injury (Perlson et al. 2005). It can then link the phosphorylated form of the MAP kinase Erk to the retrogradely trafficking injury-signaling complex. In addition, importinmediated transport to the cell body has even been speculated to underlie retrograde CREB signaling (Perry and Fainzilber 2009).

The results above are consistent with previous findings by Martin et al., who identified importins in distal neurites of Aplysia neurons as well as in the distal dendrites and axons of rodent hippocampal neurons (Thompson et al. 2004). Serotonin application at Aplysia synapses and NMDA receptor activation in mammalian neurons caused nuclear translocation of importins, suggesting a link between synapse-specific signaling and nuclear import. In a recent follow-up study, the authors provide further evidence that the nuclear transport of CREB2 from dendrites to the nucleus is mediated by importin- $\alpha$ during long-term depression (LTD), but not long-term potentiation (LTP) in both model systems (Lai et al. 2008).
A growing number of studies show that localization of specific transcripts to the axon is not only important during development, for example, by providing high local $\beta$-actin concentrations in growth cones associated with extracellular guidance cues, but also during maintenance and regeneration following nerve injury. Both lines of research, CREB and importin-mediated signaling from distal axons to the cell body, indicate that retrograde signaling based on inducible local translation constitutes an important function of RNA localization in the mature axon. Thus, it is becoming evident that deficiencies in mRNA transport to distal processes may compromise the neuron's ability to respond to injury or local stimuli within axons and dendrites (Wang et al. 2007).

\section{Linking RNA localization and dendrite morphogenesis}

As mentioned before, RNA localization to dendrites has been investigated in greater depth compared to axons, and a large number of transcripts transported to dendrites have been identified, with estimates ranging from a dozen to several hundreds (Bramham and Wells 2007). Morphological defects associated with impaired localization of dendritic RNAs mainly concern branching and the formation and maintenance of spines-mushroom-like structures at postsynaptic sites-(Hering and Sheng 2001), including subsequent physiological effects. Several lines of research tried to elucidate the functional connection between impairments in RNA sorting and the observed phenotype. One study investigated the role of brain-specific Staufen 2 (Stau2) in dendritic spine morphogenesis in mammalian neurons (Goetze et al. 2006). Staufen proteins belong to the family of double-stranded RNA binding proteins. In Drosophila, Staufen protein is required for the localization of a variety of mRNAs during oogenesis and zygotic development, e.g., oskar and bicoid RNAs (St Johnston 2005). In mammals, two distinct Staufen proteins exist arising from two different genes. Whereas Stau1 is present in many tissues, Stau2 is preferentially expressed in the brain (Wickham et al. 1999). Goetze et al. (2006) demonstrated that down-regulation of Stau2 resulted in reduced dendritic $\beta$-actin mRNA and consequently decreased $\mathrm{F}$-actin levels in cultured rat hippocampal neurons. Also, reduction in Stau2 levels led to a decrease in the number of mushroom-shaped spines but to an increase in dendritic filopodia (Fig. 1). This phenotype also coincided with a decrease in the number of synapses, showing that the effect is not restricted to the dendrites of Stau2 down-regulated neurons, but also has an impact on presynaptic sites of other cells. Since the loss of postsynaptic markers, e.g., PSD-95, is suggestive of alterations in excitatory synapses, the authors examined miniature excitatory postsynaptic currents (mEPSCs) and found an 

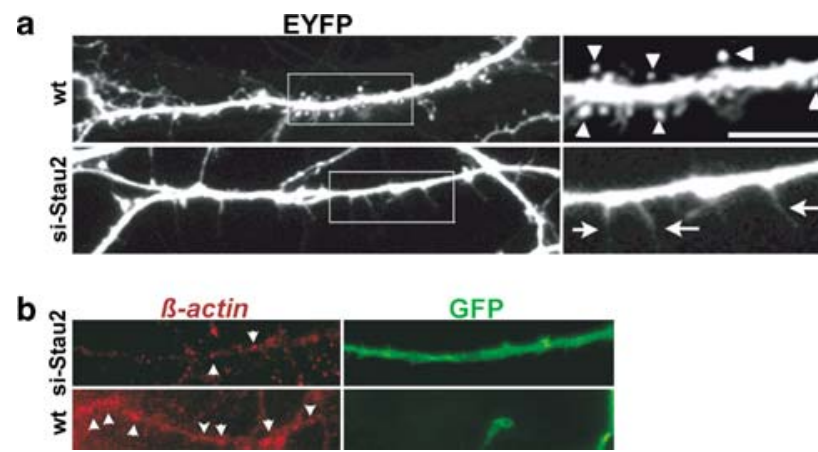

Fig. 1 Mature hippocampal neurons lacking Stau2 display significantly fewer dendritic spines. a Representative images of 15 DIV neurons either transfected with si-Stau2, a short hairpin down-regulating Stau2, or mock-treated. EYFP was used as a transfection marker. The experiment and a detailed quantification of the observed effect have been described (Goetze et al. 2006) Bar $1 \mu \mathrm{m}$. b Dendritic $\beta$-actin mRNA levels are reduced in Stau2 down-regulated hippocampal neurons. 15 DIV neurons were transfected with si-Stau2 and $\beta$-actin mRNA was visualized by FISH. In comparison, an untransfected, wild-type (wt) neuron is shown. GFP was used as a transfection marker. Pictures are reproduced with permission from (c) Goetze et al. (2006). Originally published in The Journal of Cell Biology. doi: $10.1083 /$ jcb. 200509035

attenuation of excitatory synaptic transmission due to a decreased postsynaptic responsiveness (Goetze et al. 2006). Taken together, the data suggested an important role for Stau2 in the formation and maintenance of dendritic spines in hippocampal neurons.

Similar results were found for the ubiquitously expressed Stau1 (Vessey et al. 2008). Vessey et al. found a reduction in the number of dendritic $\beta$-actin mRNPs in hippocampal neurons derived from mice carrying a loss-of-function allele for Stau1 compared to wild-type animals. They also observed reduced complexity of dendritic branching with the number of branch points being significantly lower in Stau1 loss-of-function mice. As in the case of Stau2 downregulation, dendritic spine and synapse density were reduced, while the average length of the protrusions was increased, indicating a higher proportion of long filopodia in contrast to mature mushroom-shaped dendritic spines. This supports the findings of Goetze et al. showing a coincidence of reduced dendritic $\beta$-actin mRNA levels and the aforementioned spine phenotype. Despite these observed impairments of cultured hippocampal neurons derived from Stau1 loss-of-function mice, the animals did not perform worse than their wild-type littermates in behavioral and memory tasks, but only displayed a slight reduction in locomotor activity. This possibly indicates the existence of a compensatory mechanism for the lack of functional Stau1 in vivo.

Interestingly, both studies (Goetze et al. 2006; Vessey et al. 2008) reported reduced dendritic $\beta$-actin mRNA levels and, at the same time, changes in dendrite and dendritic spine morphogenesis. Since the actin cytoskeleton is crucial for dendritic outgrowth and dendritic spine formation, maintenance and plasticity, it is reasonable to propose a functional connection between these observations. However, the exact role of Staufen proteins in this process remains elusive. In parallel, another study provided additional evidence for a synaptic function for Stau1 (Lebeau et al. 2008). The authors could define a role for Stau1 in the late phase of chemically induced LTP without affecting early phase LTP or mGluR1/5-mediated LTD of basal evoked synaptic transmission.

Another RNA-binding protein (RBP) associated with a phenotype similar to Staufen is TLS (translocated in liposarcoma). This protein is involved in splicing and nuclear export of RNA and has been observed to translocate to dendritic spines in an actin-dependent manner upon mGluR5 activation (Fujii et al. 2005). Based on the accumulation of TLS-containing granules at excitatory synapses, the authors speculate that this mechanism underlies activity-dependent translocation of RNA to the respective dendritic spines. Hippocampal neurons derived from $t l^{-1-}$ mice exhibit a significant reduction in dendritic spine number, accompanied by an increase in dendritic filopodia-like extensions. In contrast to Stau1 loss-of-function mice, the dendritic tree was more complex in TLS-deficient neurons with a higher number of primary dendrites, and more tertiary branches in distal regions. Since this phenotype cannot be attributed to TLS' ability to move into activated dendritic spines, it might be linked to its nuclear functions.

Dendritic spines are highly dynamic structures that are able to undergo changes in their size and shape upon adaptation of synaptic activity and in response to environmental stimuli and experience (Hering and Sheng 2001). Such alterations in dendritic spine number and morphology might reflect mechanisms for converting short-term adjustments in synaptic activity into lasting changes in the structure, connectivity and function of synapses. Although it is tempting to assume that dendritic spines can segregate and integrate synaptic signals, the physiological significance of spines for brain function is still unclear. Several lines of evidence suggest that dendritic RNA localization and RBPs contribute to dendritic spine morphogenesis. It is not only the studies described above that report changes in dendritic spine phenotype (upon Staufen or TLS depletion), but the alterations in the shape of dendritic protrusions have also been observed as a result of the lack of $B D N F$ mRNA in dendrites (An et al. 2008). The authors identified two polyadenylation sites in the mouse BDNF gene that give rise to distinct transcripts with a short and a long $3^{\prime}$-UTR, respectively. Only the latter localizes to dendrites, and both this short and a truncated version $\left(\mathrm{BDNF}^{\mathrm{klox}}\right)$ are restricted to the cell body, corresponding to reduced dendritic protein levels. In BDNF ${ }^{\text {klox/klox }}$ mice, Xu et al. observed an increase in dendritic spine density and a small, but highly significant 
reduction in spine head diameter as a result of a deficit in BDNF mRNA transport to dendrites (An et al. 2008). The authors conclude that the first effect is most likely due to impaired spine pruning-a process of refinement of synaptic connections, which is activity dependent-as opposed to deficits in the original formation of the protrusions. This therefore argues for a role of local BDNF in this process. Dendritic outgrowth and branching were unaffected, but electrophysiological recordings revealed a deficit in LTP. The authors hypothesize that this might result from reduced BDNF-mediated signaling leading to cytoskeletal rearrangements and neurotransmitter incorporation into the postsynaptic membrane. As such, it provides a link to the second phenotype observed, namely the smaller dendritic spine head size. On the other hand, pruning of inactive synapses is thought to be mediated by secreted pro-BDNF (Lee et al. 2001). Therefore, reduced local synthesis and secretion might explain the pruning deficit and the increased number of protrusions.

In contrast to $B D N F$, impaired dendritic localization of nanos (nos) mRNA (Fig. 2) leads to reduced terminal branch density in Drosophila class IV dendritic arborization neurons (Brechbiel and Gavis 2008). Previous work on Nos showed that it serves as a determinant for abdomen formation, and localization of its mRNA to the posterior pole of the oocyte is crucial for normal embryonic development (Lehmann and Nüsslein-Volhard 1991). The recent results by Brechbiel and Gavis suggest that the observed reduction in dendritic branching is possibly due to a defect in maintenance and not in the initial elaboration of the dendritic tree as in early larval stages no differences between wild-type and nos deficient neurons were detected. In neurons, the requirement of localization elements in the $3^{\prime}$-UTR of nos mRNA for the rescue of the mutant phenotype suggests that dendritic localization of this mRNA

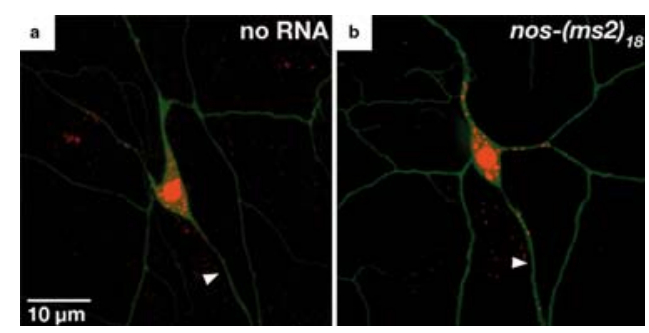

Fig. 2 Imaging of nos RNA in Drosophila peripheral larval neurons. Class IV dendritic arborization (da) neurons in semi-intact third instar larvae expressing a MCP-RFP (red) alone (control) or b MCP-RFP (red) and nos-(MS2) ${ }_{18}$ mRNA (Brechbiel and Gavis 2008); Unbound MCP-RFP is sequestered in the nucleus due to a nuclear localization signal (NLS). Arrowheads indicate the axon. Neurons are filled with GFP (green). Pictures are reproduced with permission from (C) Brechbiel and Gavis (2008). Originally published in Current Biology. doi:10.1016/j.cub.2008.04.033 mediates its presumed function in maintenance of dendritic branching. Interestingly, increased nos translation also leads to the same phenotype as impaired dendritic localization. Brechbiel and Gavis showed that mutations in the repressors or their binding sites in the nos 3'-UTR, which in the oocyte serve to prevent ectopic synthesis and restrict the gene product to its designated site of action, also caused a reduction in the number of terminal dendritic branches. The authors thus conclude that apart from the localization of the RNA, translational repression is also crucial for normal dendrite morphogenesis and needs to be tightly regulated.

In summary, several studies have shown general effects resulting from impairments in the localization of certain transcripts. On the one hand, the shape of dendritic spines is often altered and the plethora of causes associated with this phenotype presents it as an indicator of abnormalities in dendritic regulatory mechanisms or structural organization. The other common feature of neurons with a deficit in RNA localization appears to be alterations in dendritic branching. For most of the cases, however, a model linking reduced local translation and protein concentration-which are the logical consequences of impaired RNA localization associated with the observed phenotypes - and the defects in dendrite branching and spine morphology is still lacking.

\section{Potential roles of RNAs at the synapse}

The mouse knock-out of the $\alpha$-subunit of the calcium/ calmodulin-dependent protein kinase II (CaMKII $\alpha$ ) opened a whole new field in neuroscience when it was systematically used to study learning and memory (Elgersma et al. 2004). Already in 2002, Mayford et al. generated another interesting mouse mutant by genetically disrupting the 3 '-UTR of CaMKII $\alpha$ thus interfering with its dendritic localization to synapses. This led to a significant reduction in the level of CaMKII $\alpha$ at postsynaptic sites of neurons. Subsequently, these transgenic mice were used to investigate the physiological importance of RNA localization and local CaMKII protein synthesis (Miller et al. 2002). These animals exhibited reduced late-phase LTP and impairments in memory tasks, e.g., the Morris water maze and contextual fear conditioning. Mayford et al. concluded from their study that local translation of CaMKII $\alpha$ is not required for induction of LTP, but instead for its maintenance as well as the establishment of long-term hippocampal-dependent memories. Since the modified CaMKII $\alpha$ mRNA lacking the dendritic targeting signal was present throughout development, their work could not distinguish whether the observed impairments reflected an acute requirement for CaMKII $\alpha$ synthesis versus a constitutive, long-term effect of synaptic CaMKII $\alpha$. Nonetheless, this study provided the first intriguing 
experimental evidence that dendritic RNA localization is physiologically important for synapse function.

Another well-studied dendritically localized mRNA encodes the activity-regulated cytoskeleton-associated protein (Arc or Arg3.1) (Link et al. 1995; Lyford et al. 1995). In contrast to other proteins derived from dendritically localized mRNAs, Arc expression is rapidly induced by synaptic activity, e.g., seizures, LTP and memory-related memory tasks (Tzingounis and Nicoll 2006). Consequently, Arc expression has served as a premier marker to map neuronal networks that underlie information processing and plasticity. Newly synthesized Arc mRNA is rapidly delivered to dendrites and accumulates selectively near activated synapses, following synaptic activity (Steward and Worley 2001). This targeting process requires NMDA receptor activation. Kuhl et al. generated an Arc knock-out mouse and showed that the mutant mice have severe memory deficits, as they do not form either long-term spatial, fear or taste memories. Furthermore, Arc also regulates orientation selectivity in the visual cortex (Wang et al. 2006). It is a remarkable property of neurons in the visual cortex to be able to detect local bars and edges in the processed visual images and to encode their orientations (Hubel and Wiesel 1962).

How does Arc affect the formation of memories? A series of studies by three laboratories, all published backto-back in Neuron, provided the first important insight into this exciting topic (Chowdhury et al. 2006; Plath et al. 2006; Rial Verde et al. 2006; Shepherd et al. 2006). The cited papers provided the first compelling link between Arc and AMPA receptor endocytosis, synaptic plasticity and memory function. First, Arc over-expression or knockdown critically influenced AMPA receptor surface expression, and abolished synaptic homeostasis. These findings suggest that Arc needs to be dynamically expressed as it critically controls synaptic strength and cellular excitability. Second, over-expression of Arc induced endocytosis of AMPA receptors and occluded LTD. Third, Arc knock-out mice failed to form long-lasting memories for implicit and explicit learning tasks, despite intact short-term memory. This suggests that Arc plays an important role in memory retention. In contrast to CaMKII $\alpha$ and MAP2 mRNAs, the dendritic targeting element of Arc mRNA has not yet been unambiguously identified. It is clear, however, that the $A r c$ 3'-UTR enhances translation upon BDNF stimulation (Rao et al. 2006), but it has not yet been possible to dissect the contribution of dendritic targeting of Arc mRNA to dendritic spine morphogenesis as in the case of CaMKII $\alpha$ (Miller et al. 2002, see above).

Taken together, these studies on CaMKII $\alpha$ and Arc mRNAs convincingly show that dendritic RNA localization and subsequent local protein synthesis at activated synapses is of great physiological relevance and critically contributes to both synaptic plasticity and learning and memory. To exert these important functions, dendritic RNA transport and translational control need to be intimately linked and tightly regulated (Dahm and Kiebler 2005; Hüttelmaier et al. 2005; Richter and Klann 2009). During transport of localized transcripts within RNPs to synapses, translation appears to be inhibited (Kiebler and Bassell 2006). Only upon synaptic activation, translation is activated thereby giving rise to synapse-specific modifications and thus to several forms of long-lasting hippocampal synaptic plasticity. Furthermore, translational control at the synapse must be an extraordinarily fine-tuned process (Costa-Mattioli et al. 2009; Richter and Klann 2009). Several key mechanisms have been proposed for this process. First, initiation factors of translation, e.g., eIF4E and eIF4E-binding protein 2 (4E-BP2), are regulated by key kinases, e.g., mammalian targets of rapamycin (mTOR), S6 kinase and Mnk1. Second, selected RBPs, e.g., FMRP, Pumilio2, Zipcode-binding protein 1 (ZBP1) and many others, act as translational regulators at the synapse, tightly repressing translation. Third, another sequence-specific RBP, termed cytoplasmic polyadenylation element binding protein (CPEB) stimulates translation by inducing cytoplasmic poly(A) elongation of several mRNAs in neurons in response to synaptic activity (Richter and Klann 2009). Fourth, members of the exon junction complex (EJC), e.g., eIF4AIII, Barentsz/MLN51, Y14, Magoh, appear to regulate synaptic strength (Giorgi et al. 2007; Richter and Klann 2009). Fifth, there is recent evidence that small non-coding RNAs might exert translational control at the synapse providing yet another attractive mechanism to prevent unwanted, premature local protein synthesis (see below). In conclusion, it does not come as a surprise to learn that many alterations in the mentioned processes yield synaptic dysfunction and diseases (Sonenberg and Hinnebusch 2007, 2009).

\section{Small RNAs at the synapse}

In the previous section, we described the key finding implicating dendritic localization of mouse CaMKII $\alpha$ mRNA with synaptic plasticity. This is beautifully corroborated by a remarkable study that showed for the first time that protein synthesis at the synapse is responsible for the formation of long-lasting memory in Drosophila (Ashraf et al. 2006). In their study, Kunes et al. took advantage of the power of fly genetics combined with an olfactory learning assay. They investigated antennal lobe projection neurons, the first-order interneurons in the olfactory sensory pathway of Drosophila, and showed that dendritic expression of CaMKII is regulated by its $3^{\prime}$-UTR and stimulated by neural activity including conditioned olfactory learning. 
Furthermore, the authors were able to convincingly demonstrate in flies that were defective in factors important for the function or assembly of the RNA-induced silencing complex (RISC), that synaptic expression of CaMKII is negatively regulated by the RISC pathway (Ashraf et al. 2006). They identified potential binding sites for two miRNAs, miR-280 and miR-289, in the 3'-UTR of CaMKII RNA. These sites were similar to those mRNAs in the 3'-UTRs of oskar and kinesin heavy chain RNA, both of which are affected in RISC mutants. The authors investigated the role of RISC in CaMKII expression. Armitage, an RNA helicase involved in RISC function, was degraded in a proteasome-dependent manner at particular synapses, which allowed protein synthesis to occur. Importantly, Armitagemutant flies show impaired long-term memory. In conclusion, Kunes et al. proposed the following model: signals leading to the formation of long-lasting memories activate the proteasome to degrade Armitage. This allows the expression of CaMKII and other proteins causing synaptic plasticity and long-term memory in Drosophila. These findings are nicely complemented by a recent study by Kosik et al. in neurons on the mammalian ortholog of Armitage, Mov10. Mov10 is an RNA helicase that associates with Argonaute proteins, among others, in mammalian cells (Meister et al. 2005). In the new study, Mov10 is found to be degraded via the proteasome pathway in response to NMDA receptor activation (Banerjee et al. 2010).

Recent work by Schratt et al. provided further evidence that miRNAs regulate synaptic protein synthesis (Schratt et al. 2006; Siegel et al. 2009). In one study, the authors identified a miRNA, miR-134, which localized at synaptic sites (Schratt et al. 2006), where it partially inhibited translation of Lim-domain-containing protein kinase 1 (Limkl) mRNA by binding to a single binding site in its $3^{\prime}$-UTR . Limk1 is of particular interest in this context as it regulates actin filament dynamics through inhibition of ADF/cofilin. Furthermore, Limk1 knockout mice showed abnormalities in dendritic spine structure similar to those observed upon miR-134 over-expression (Meng et al. 2002). It is significant that stimulation with BDNF relieved miR-134-dependent repression of Limkl translation, resulting in the growth of dendritic spines. This relief of repression involved the BDNF receptor TrkB and mTOR. The authors propose that a miRNA may act locally at individual synapses on dendritically localized mRNAs, e.g., Limkl mRNA, thereby contributing to synapse-specific modifications. This in turn would allow regulation of dendritic spine morphogenesis and thereby modulation of synaptic strength.

In a more recent study, Schratt, Martinez et al. identified another activity-regulated miRNA that also localizes to synapses (Siegel et al. 2009). As in the case of miR-134, alterations of miR-138 levels led to a similar dendritic spine phenotype. As can be inferred from reduced dendritic spine size, the authors found that over-expression of miR-138 led to a decrease in AMPA receptor cluster size and mEPSCs, which are typically mediated by these receptors. A computational approach identified a number of potential target mRNAs, one of which, Acyl protein thioesterase 1 (APT1), was validated in this study as a miR-138 target in neurons. Interestingly, APT1 is an enzyme that catalyzes the depalmitoylation of target proteins, however, its function in neurons is unknown. Notably, palmitoylation has also been implicated in the regulation of synaptic plasticity (El-Husseini et al. 2002). APT1 could therefore critically regulate membrane anchoring of many postsynaptic proteins at the synapse. The authors focused on a previously identified substrate of APT1, the G protein subunit $\mathrm{G} \alpha 13$, which is a known RhoA activator. Down-regulation of APT1 via RNAi or expression of membrane-localized $\mathrm{G} \alpha 13$ both led to a decrease in the size of dendritic spines thereby counteracting dendritic spine enlargement caused by miR-138 inhibition. Taken together, these findings indicate that a possible miR-138 function might be repression of APT1-regulated depalmitoylation of $\mathrm{G} \alpha 13$ at the synapse. This is the first study to suggest a causal relationship between a miRNA and post-translational modification of synaptic proteins yielding changes in dendritic spine morphology. It will be interesting to learn whether miR-138, like miR-134, is regulated by synaptic activity and which additional targets are regulated by both miRNAs at the synapse. However, recent evidence (Davis et al. 2008) may indicate more widespread effects of small RNAs at the synapse, since conditional loss of the pre-miRNA processing enzyme Dicer in excitatory forebrain neurons of mice results in microcephaly, reduced dendritic branch elaboration and even larger increases in dendritic spine length than the one reported by Schratt et al.

In the nervous system, the vast majority of the known miRNAs are expressed where they might exert a diverse range of functions affecting a large number of genes (Kosik 2006). These can range from neural patterning, establishment and maintenance of cell identity, axonal pathfinding, and apoptosis to synaptic plasticity in mature neurons, and implicate miRNAs in nervous system diseases. As outlined above, a particularly attractive neuronal function for miRNA-mediated regulation is local translational control associated with synaptic plasticity. However, there are only few studies providing convincing experimental evidence for such a model (Ashraf et al. 2006; Schratt et al. 2006; Siegel et al. 2009). As it is the case with any rapidly evolving field, demonstrating the presence of miRNAs at the synapse raises more questions than answers and suggests directions for future research. First, based on the presented data, different models have been postulated. A synaptic signal causes a change from translational inhibition to activation, while the miRNA may remain associated with 
translationally activated mRNA on polysomes (Schratt et al. 2006). Alternatively, it has been proposed that activation of translation would result from the release of mRNA from a translational silencing complex, the RNA granule, to polysomes (Kosik 2006). A third possibility for miRNAmediated repression is a possible role of processing bodies (P-bodies) at the synapse (Zeitelhofer et al. 2008). It will be of crucial importance to determine the precise contributions of those distinct cellular structures to miRNA-dependent repression of translation and, furthermore, to understand how these synaptic signals relieve translational repression mechanistically.

Finally, the question remains how certain miRNAs reach the synapse and others are excluded from it. Do those miRNAs piggy-back on a target mRNA that contains dendritic localization elements, or alternatively, are dendritic miRNAs to be processed from pre-miRNAs at the synapse and meet their target mRNA(s) only there? Do key RBPs that preferentially bind to $3^{\prime}$-UTRs of dendritically localized transcripts act as modifiers of miRNA-mediated repression, as recently suggested by Witold Filipowicz (Filipowicz et al. 2008)? RBPs including HuR, FXR1 and Dead end 1 (reviewed in Filipowicz et al. 2008) have been proposed to exert such regulatory functions, however, none of them has yet been shown to mediate or modify miRNA repression at the synapse.

\section{Real-time imaging of RNA as a means to investigate the molecular mechanisms of dendritic RNA transport}

The investigation of the mechanisms underlying RNA transport and localization has been greatly advanced by the application of technologies that allow the visualization of RNA in living cells. We will introduce some of the most widely used technologies for real-time imaging of RNA (Fig. 3) and outline their great potential to unravel how mRNA is transported to its final destination in primary neurons.

To get first insight into the dynamics of RNA transport in a living neuron, green fluorescent protein (GFP)-tagged versions of a series of RBPs, e.g., Stau1, Stau2, Barentsz, ZBP1, CPEB, FMRP, Pur $\alpha$, TLS, have been transiently expressed in neurons (Köhrmann et al. 1999; Huang et al. 2003; Oleynikov and Singer 2003; Kanai et al. 2004; Dictenberg et al. 2008; Zeitelhofer et al. 2008). In most cases, the formed RNPs were transported along microtubules into dendrites. However, it was not demonstrated at the time that the over-expressed RBPs are associated with their target mRNAs and that the observed particles indeed contain the RNA of interest.

A number of research groups have now succeeded in combining GFP technology with key advances in RNA biochemistry to directly monitor RNA localization in living cells and in real time (reviewed in Brodsky and Silver 2002). In general, these systems have two components: (i) an RBP-GFP fusion and (ii) a generic RNA including RBP binding sites. When both constructs are co-expressed in the same cell, the two will interact and allow the RNA to be conveniently monitored by fluorescence microscopy in real time. Although there is the potential danger to over-express the RNA of interest, causing aberrant localization, expression levels can often be adjusted. Two RNA hairpin-RBP interactions have been successfully applied for GFP RNA imaging: the bacteriophage MS2 capsid protein, by Rob Singer et al. in yeast (Bertrand et al. 1998); and the human splicing protein U1A (Brodsky and Silver 2000). Both proteins bind the corresponding small stem-loops with very high affinity. In the case of the MS2 system (Fig. 3), several key improvements have been applied. First, multiple repeats of the MS2 stem-loops have been incorporated into the RNA sequence of interest to sequester multiple MS2 proteins each fused to GFP. Subsequent work by the Singer lab showed that 24 repeats were required to detect single mRNA molecules and to visualize individual mRNP complexes in living cells (Fusco et al. 2003). Second, the Singer lab was able to significantly reduce the noise from free GFP molecules within the cytoplasm by adding a NLS to the MS2-GFP protein. This sequesters the resulting fusion protein within the nucleus when not bound to an MS2-containing RNA target. The MS2 system has been used routinely for monitoring RNA transport and anchoring during Drosophila oogenesis and early embryogenesis providing important insight into the localization mechanism of the axis determinants nos (Forrest and Gavis 2003), bicoid (Weil et al. 2006; Weil et al. 2008), gurken (Jaramillo et al. 2008) and oskar RNA (Zimyanin et al. 2008).

The first group to employ the MS2-GFP system in the nervous system was Kosik et al. (Rook et al. 2000), who successfully visualized CaMKII $\alpha$ mRNA fused to eight copies of the MS2 binding element. The authors observed movement of discrete RNA granules throughout the dendrites of transfected cells and the first insight into their dynamics was gained. Interestingly, depolarization increased the number of RNA granules in dendrites.

Gary Bassell et al. (Dictenberg et al. 2008) used the same approach to investigate the role of the fragile $\mathrm{X}$ mental retardation protein (FMRP) in dendritic RNA transport (Bassell and Warren 2008). While a large body of evidence points to a role of FMRP in translational regulation, the involvement for FMRP in mRNA localization had not-to this point—been directly addressed. In this study, mRNAGFP particles were tracked in hippocampal neurons derived from either wild-type or FMRP KO mice, in the presence of an mGluR1 agonist. Interestingly, striking differences in the motility of the observed CaMKII $\alpha$ mRNA particles were found. Whereas $23 \%$ of all particles were motile in wild-type 

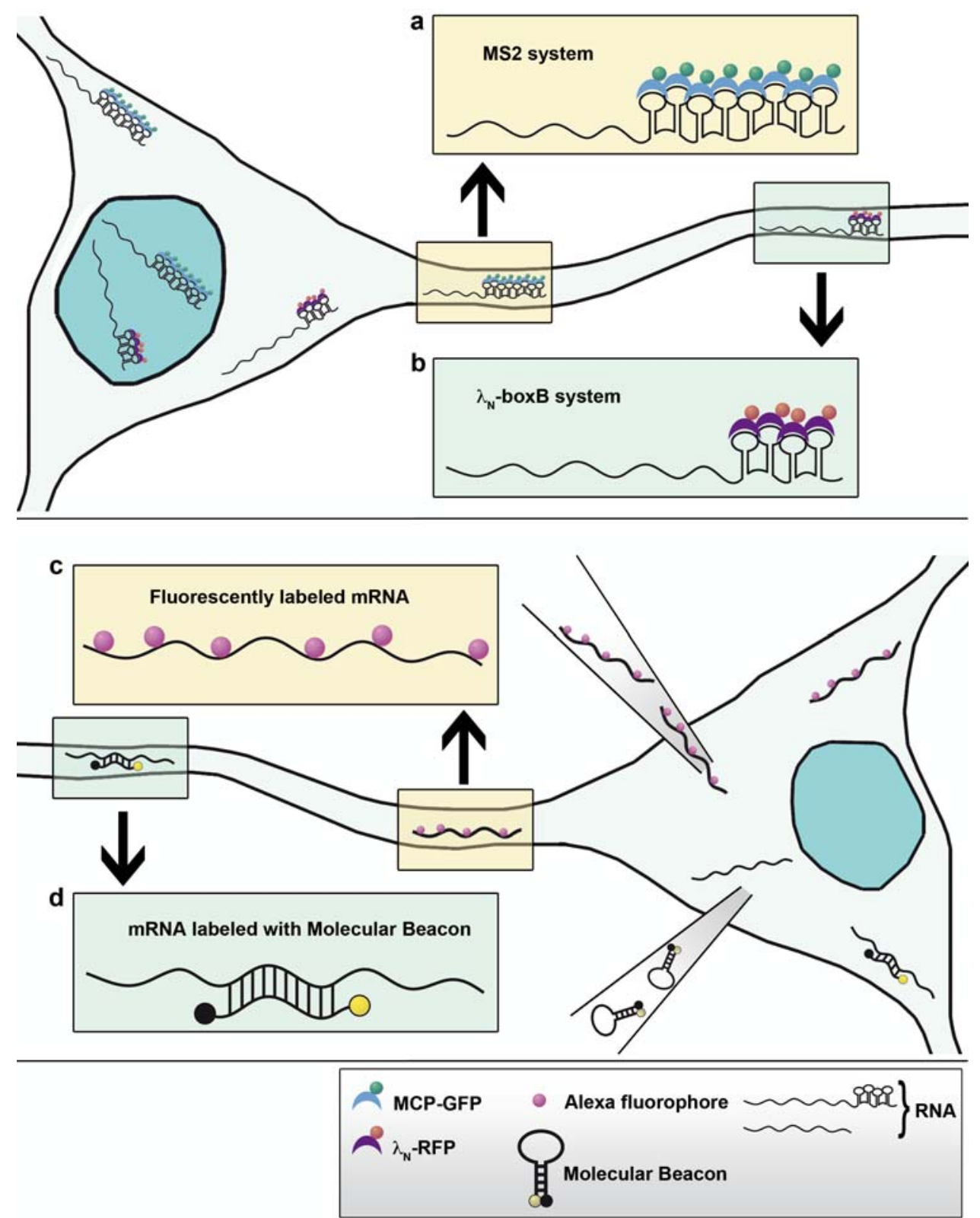

Fig. 3 Approaches for real-time visualization of RNA transport in neurons. a The MS2 system takes advantage of the specific interaction between the bacteriophage MS2 coat protein (MCP) and its cognate RNA binding sites. The RNA of interest is tagged with multiple MS2 binding sites and is transiently transfected in neurons together with MCP fused to GFP (or other fluorescent proteins). The RNA tethers GFP molecules in the nucleus and the complex gets exported to the cytoplasm and travels toward dendrites or axons. $\mathbf{b}$ The $\lambda_{\mathrm{N}}$-boxB system uses the stable association between a peptide derived from a phage $\lambda$ protein $\left(\lambda_{\mathrm{N} 22}\right)$ and a small hairpin (boxB), to label RNA molecules with fluorescent proteins (RFP is shown here). Employment of this

neurons, only $5 \%$ of mRNA particles exhibited directed movements in FMRP KO neurons (Dictenberg et al. 2008). By taking advantage of a dominant-negative approach by over-expressing the KLC-binding, C-terminal domain of FMRP, they could convincingly demonstrate a significant approach in neurons, in combination with the MS2 system would allow simultaneous detection of two transcripts in neurites. c Alexa-labeled UTPs are incorporated in the RNA of interest during in vitro transcription. Upon injection into the cell body of neurons, the labeled transcript recruits the transport machinery and localizes to neuronal processes. d Molecular beacons are stem-loop oligonucleotides bearing a fluorophore and a quencher at either end. When not bound to their target sequence, the hairpin keeps the quencher in the vicinity of the dye. Formation of hybrids between MBs and native transcripts, upon injection of MBs into the cytoplasm of neurons restores fluorescence and labels the target RNA

reduction of FMRP particles in wild-type neurons as well as a significant increase in the length and number of dendritic protrusions. This effect parallels previous findings in the mouse model and in humans with fragile $\mathrm{X}$ syndrome. According to the model the authors propose, changes in the 
activity-dependent synaptic localization and transport dynamics of FMRP target mRNAs involved in synaptogenesis and plasticity may contribute to translational and synaptic defects observed in fragile $\mathrm{X}$ syndrome.

In Drosophila larval sensory neurons, Nos is required for the maintenance of dendritic complexity (Brechbiel and Gavis 2008). Expression of a nos transgene tagged with 18xMS2 binding sites allowed sensitive detection of nos particles in the processes of class IV dendritic arborization neurons of intact larvae (see above), providing the first evidence for dendritic localization of nos RNA (Fig. 2; Brechbiel and Gavis 2008). Deletion analysis showed that localization of nos in the PNS is mediated by the same elements that direct the maternal transcript to the posterior pole of the oocyte. In addition, live imaging of nos particles at high resolution identified fast anterograde and retrograde motion, suggesting motor-dependent transport of the transcript along microtubules.

A similar RNA reporter system (Fig. 3) is composed by a fluorescent protein fusion of a 22 amino acid peptide derived from a phage $\lambda$ protein $\left(\lambda_{\mathrm{N} 22}\right)$ and a 15 nt hairpin (boxB), to which $\lambda_{\mathrm{N} 22}$ binds stably and specifically (Daigle and Ellenberg 2007). BoxB, like MS2 motifs, can be introduced in any transcript, thus mediating tethering of the peptide. Combination of the MS2 and $\lambda_{\mathrm{N}}$-boxB systems was employed for simultaneous, dual color imaging of two different yeast RNAs and revealed a high degree of coordination of their trafficking to the bud of $S$. cerevisiae (Lange et al. 2008).

Several other advanced methods for direct imaging of mRNAs have been developed recently (Fig. 3). In one case, RNA labeled with fluorescent dyes is injected into living cells and monitored in real-time. The RNA of interest is synthesized in vitro in the presence of Alexa-labeled UTP. This approach offers several advantages. The signal to noise ratio is usually high, as free Alexa nucleotides are removed prior to injection and imaging takes place soon after injection, before any degradation occurs. The high sensitivity of the method allows a very fast acquisition rate, which is necessary for the tracking of fast moving RNA particles and detailed analysis of their motility. Furthermore, cytoplasmic injection of fluorescently labeled RNA has been instrumental for unraveling the mechanism of RNA localization. For example, injection of truncated transcripts can reveal the signals necessary and sufficient for localization and injection of RNAs in mutants, co-injection with antibodies and other inhibitors can identify the molecular motors and other key proteins involved in RNA transport. Finally, the method allows simultaneous imaging of two or more RNAs, or RNA-miRNA pairs upon simultaneous or successive injection in the cytoplasm of differentially labeled transcripts.

Davis et al. routinely employed injection of Alexalabeled RNA to visualize transport intermediates of pair rule transcripts from the site of injection to the apical cytoplasm of Drosophila embryos (Wilkie and Davis 2001), as well as maternal transcripts within the fly oocyte, or from the nurse cells toward the oocyte (MacDougall et al. 2003; Clark et al. 2007). In all cases, fast time-lapse imaging combined with high spatial resolution revealed rapid, directed movements of RNA particles that were microtubule- and dynein-dependent, as demonstrated by inhibition or loss-of-function studies. The assay was also fundamental for further dissection of the transport machinery, for example the localization signals that direct the RNA (Bullock et al. 2003; Van De Bor et al. 2005) and the components that link them to the molecular motors (Bullock et al. 2006).

Application of the above approach to neurons has not been straightforward. The complications arise from the method of delivery of the RNA into the cell. Neurons are very sensitive to changes in their microenvironment and physical manipulation (Washbourne and McAllister 2002), minimizing viability after microinjection. For example, the majority of mammalian neurons are unhealthy or dying upon injection, as shown by their overall morphology, their detachment from their substrate or formation of granules in the cell body (Lappe-Siefke et al. 2008). Survival rates are highly dependent on the quality of the neuronal culture and at least for mammalian neurons, preparation of optimal cultures is not always a straightforward task. Low injection pressure and short injection times constitute other critical parameters and recent advances now allow automated, controlled microinjection. However, the drawback remains that fluorescently labeled RNA usually needs to be introduced in the cell at much higher amounts than other materials, such as plasmid DNA, siRNAs or antibodies, making optimization of injection settings difficult and limiting cell viability. Alternatively, less invasive methods of introduction of RNA in neurons, such as cellular uptake, lipid-based transfection or viral delivery, could be certainly advantageous over cytoplasmic injection. However, due to requirement for large quantity of RNA per cell, these techniques are unlikely to be suitable for real-time imaging of RNA in living neurons.

Despite these limitations, microinjection of Alexalabeled RNA has been successfully applied to rat hippocampal neurons in culture, in three recent independent studies (Shan et al. 2003; Gao et al. 2008; Tuebing et al. 2010). In the first report, Smith et al. showed that the localization of a microinjected, chimeric RNA reporter depends on the presence of an A2RE (heterogeneous nuclear ribonucleoprotein A2 response element), shown before for Myelin Basic Protein (MBP) RNA of oligodendrocytes (Ainger et al. 1993, 1997), and on its recognition by heterogeneous nuclear ribonucleoprotein A2 (hnRNPA2). In another recent study (Gao et al. 2008), the assay was employed to 
further elucidate the mechanism of dendritic localization by the A2 pathway. The authors demonstrated that CaMKIIa, Neurogranin $(N G)$ and Arc RNAs all bear cis-acting A2REs. The transcripts were shown to co-assemble in the same dendritic granules, recruit hnRNPA2 and to be targeted to dendrites in an hnRNPA2-dependent manner. In the third study (Tuebing et al. 2010) microinjection of CaMKIIa and subsequent real-time imaging at high temporal resolution identified fast bidirectional motility of the transcript along dendrites. Furthermore, simultaneous injection of differentially labeled MAP2 and CaMKIIa showed that they were sorted into distinct RNPs, arguing that at least these transcripts are targeted to dendrites by independent pathways. Another promising application of this approach is the simultaneous imaging of miRNAmRNA pairs at the synapse. As previously referred, it was recently shown that miR-134 localizes at synaptic sites where it is thought to repress the translation of LimK1, thus regulating dendritic spine morphogenesis (Schratt et al. 2006). Schratt et al. co-injected in hippocampal neurons miR-134 together with LimK1, and demonstrated that the two RNAs co-localize in the same RNPs within dendrites. This interaction is specific, since it is abolished by mutations at the miR-134 binding site in the 3'-UTR of LimK1 RNA. Co-visualization of miR-134 and Limkl was the first attempt to detect a miRNA together with its target mRNA at synapses. Similar experiments can be instrumental in the future for the validation of miRNA-mRNA interactions in vivo, as the double detection of the native neuronal transcripts is currently not feasible.

Another promising strategy to visualize RNA introduces an exogenous probe called molecular beacon (MB) in living cells, in order to follow the distribution and dynamics of native RNA in real time (Fig. 3). MBs are stem-loop shaped oligonucleotides designed so that they fluoresce only upon hybridization to their target sequence. The hairpin structure-resulting from complementary sequences on either end of the probe-brings a quencher close to the fluorophore. Hybridization disrupts this configuration, thus restoring the fluorescent signal (Bratu et al. 2003). Several criteria need to be met for MBs to be successfully used to monitor RNA transport in living cells. Accessibility of the target sequence is one requirement, as parts of the RNAs are already paired in secondary or higher order structures or masked by proteins. It is also important that MBs do not disrupt the conformation of the target RNA upon binding and therefore interfere with its localization. Finally, the probes should be highly specific for their target and not recognize any other transcripts in the cell.

Molecular beacons have been used to visualize oskar mRNA during its entire path, from the nurse cells to the posterior of the Drosophila oocyte (Bratu et al. 2003). A follow-up study (Mhlanga et al. 2009) used a cocktail of four MBs, each targeting a different region of oskar. This approach significantly enhanced sensitivity and allowed fast, 3D imaging of individual native oskar mRNPs and detailed analysis of their kinetics, reshaping and interaction with Staufen during their multi-step transport. An alternative strategy to increase the intensity and reduce background signal of MBs was employed by Tyagi et al. Engineering of 96 tandem repeats of the beacon target sequence in a reporter RNA resulted in single molecule sensitivity (Vargas et al. 2005).

The employment of MBs for direct visualization of mRNA in neuronal cells is, however, less straightforward. For reasons mentioned above, the delivery of the probes in living neurons by microinjection is challenging. Conventional transfection methods have been tested and proven inefficient and not fast or direct enough to prevent degradation of the molecular beacon in the transfection medium (Nitin et al. 2004). The authors demonstrated that modified, peptide-linked MBs are instead self-delivered into human dermal fibroblasts offering a promising possibility for application of the method in living neurons.

Multiply labeled tetravalent RNA imaging probes (MTRIPs) were recently developed and utilized to image RNA in hRSV (human respiratory syncytial virus), cancer cells and primary fibroblasts (Santangelo et al. 2009). The probes consist of four, linear nucleic acids labeled with multiple high quantum-yield fluorophores, linked together by biotin-streptavidin. They are delivered in the cell by reversible membrane permeabilization with streptolysin $\mathrm{O}$, a treatment that is detrimental to neurons. Therefore, the establishment of alternative methods of delivery of MTRIPs will be necessary for the application of this approach to the study of RNA localization in axons and dendrites.

\section{Outlook/Future experiments}

In this review, we highlighted a set of new experiments implicating RNA localization in neurons with neurotrophin signaling, axon outgrowth, dendrite and dendritic spine morphogenesis, as well as with synaptic plasticity. In addition, we outlined the role of microRNAs in translational control in dendrites and its implications for learning and memory. Furthermore, it is worth to note that the RNAi machinery is also present and active within axons at distal sites (Hengst et al. 2006). It will be interesting in the future to learn about the role of the RNAi machinery in axons.

The development of approaches for real-time imaging of RNA by the laboratories of Gary Bassell, Simon Bullock, Ilan Davis, Liz Gavis, Daniel St Johnston and Rob Singer have greatly advanced our understanding of RNA localization in mechanistic terms (Bullock and Ish-Horowicz 2001; Wilkie and Davis 2001; Brechbiel and Gavis 2008; Zimyanin 
et al. 2008). It will be particularly interesting to study the mechanism of dendritic or axonal targeting of neuronal transcripts. Which molecular motors are involved? Which RBPs are necessary for the delivery of certain RNAs to growth cones or synapses? Do different dendritically localized transcripts share the same transport machinery or are they sorted into distinct RNPs? What signal(s) cause(s) their unpacking at the synapse and their local translation upon synaptic activation? As outlined above, several new and exciting methods of visualizing RNA exist that are now being applied to neurons. We anticipate that these approaches will not only yield a wealth of new information, but ultimately will provide a consensus on how RNA localization works mechanistically. They will potentially also help to shed light on the functional basis of morphological deficits in neurons with impairments in RNA trafficking and to elucidate the molecular events underlying learning and memory.

Acknowledgments The authors apologize for omitted references due to space restraints. The authors would like to thank Jacki Heraud, Alexandre Raposo and Lucia Schoderböck for critical comments and Sebastian Butter for assistance in preparing the figures. Support comes from the FWF, the RNA quality programme (Eurocores, ESF) and the HFSP.

Open Access This article is distributed under the terms of the Creative Commons Attribution Noncommercial License which permits any noncommercial use, distribution, and reproduction in any medium, provided the original author(s) and source are credited.

\section{References}

Ainger K, Avossa D, Morgan F, Hill SJ, Barry C, Barbarese E, Carson JH (1993) Transport and localization of exogenous myelin basic protein mRNA microinjected into oligodendrocytes. J Cell Biol 123:431-441

Ainger K, Avossa D, Diana AS, Barry C, Barbarese E, Carson JH (1997) Transport and localization elements in myelin basic protein mRNA. J Cell Biol 138:1077-1087

An JJ, Gharami K, Liao GY, Woo NH, Lau AG, Vanevski F, Torre ER, Jones KR, Feng Y, Lu B, Xu B (2008) Distinct role of long 3' UTR BDNF mRNA in spine morphology and synaptic plasticity in hippocampal neurons. Cell 134:175-187

Ando R, Hama H, Yamamoto-Hino M, Mizuno H, Miyawaki A (2002) An optical marker based on the UV-induced green-to-red photoconversion of a fluorescent protein. Proc Natl Acad Sci USA 99:12651-12656

Arimura N, Kaibuchi K (2007) Neuronal polarity: from extracellular signals to intracellular mechanisms. Nat Rev Neurosci 8:194205

Ashraf SI, McLoon AL, Sclarsic SM, Kunes S (2006) Synaptic protein synthesis associated with memory is regulated by the RISC pathway in Drosophila. Cell 124:191-205

Banerjee S, Neveu P, Kosik KS (2009) A coordinated local translational control point at the synapse involving relief from silencing and MOV10 degradation. Neuron 64:871-884

Barnes AP, Polleux F (2009) Establishment of axon-dendrite polarity in developing neurons. Annu Rev Neurosci 32:347-381
Bassell GJ, Warren ST (2008) Fragile X syndrome: loss of local mRNA regulation alters synaptic development and function. Neuron 60:201-214

Bertrand E, Chartrand P, Schaefer M, Shenoy SM, Singer RH, Long RM (1998) Localization of ASH1 mRNA particles in living yeast. Mol Cell 2:437-445

Bramham CR, Wells DG (2007) Dendritic mRNA: transport, translation and function. Nat Rev Neurosci 8:776-789

Bratu DP, Cha BJ, Mhlanga MM, Kramer FR, Tyagi S (2003) Visualizing the distribution and transport of mRNAs in living cells. Proc Natl Acad Sci USA 100:13308-13313

Brechbiel JL, Gavis ER (2008) Spatial regulation of nanos is required for its function in dendrite morphogenesis. Curr Biol 18:745-750

Brodsky AS, Silver PA (2000) Pre-mRNA processing factors are required for nuclear export. RNA 6:1737-1749

Brodsky AS, Silver PA (2002) Identifying proteins that affect mRNA localization in living cells. Methods 26:151-155

Bullock SL, Ish-Horowicz D (2001) Conserved signals and machinery for RNA transport in Drosophila oogenesis and embryogenesis. Nature 414:611-616

Bullock SL, Zicha D, Ish-Horowicz D (2003) The Drosophila hairy RNA localization signal modulates the kinetics of cytoplasmic mRNA transport. EMBO J 22:2484-2494

Bullock SL, Nicol A, Gross SP, Zicha D (2006) Guidance of bidirectional motor complexes by mRNA cargoes through control of dynein number and activity. Curr Biol 16:1447-1452

Chowdhury S, Shepherd JD, Okuno H, Lyford G, Petralia RS, Plath N, Kuhl D, Huganir RL, Worley PF (2006) Arc/Arg3.1 interacts with the endocytic machinery to regulate AMPA receptor trafficking. Neuron 52:445-459

Clark A, Meignin C, Davis I (2007) A Dynein-dependent shortcut rapidly delivers axis determination transcripts into the Drosophila oocyte. Development 134:1955-1965

Costa-Mattioli M, Sossin WS, Klann E, Sonenberg N (2009) Translational control of long-lasting synaptic plasticity and memory. Neuron 61:10-26

Cox LJ, Hengst U, Gurskaya NG, Lukyanov KA, Jaffrey SR (2008) Intra-axonal translation and retrograde trafficking of CREB promotes neuronal survival. Nat Cell Biol 10:149-159

Dahm R, Kiebler M (2005) Cell biology: silenced RNA on the move. Nature 438:432-435

Daigle N, Ellenberg J (2007) LambdaN-GFP: an RNA reporter system for live-cell imaging. Nat Methods 4:633-636

Davis TH, Cuellar TL, Koch SM, Barker AJ, Harfe BD, McManus MT, Ullian EM (2008) Conditional loss of Dicer disrupts cellular and tissue morphogenesis in the cortex and hippocampus. J Neurosci 28:4322-4330

Dictenberg JB, Swanger SA, Antar LN, Singer RH, Bassell GJ (2008) A direct role for FMRP in activity-dependent dendritic mRNA transport links filopodial-spine morphogenesis to fragile $\mathrm{X}$ syndrome. Dev Cell 14:926-939

Elgersma Y, Sweatt JD, Giese KP (2004) Mouse genetic approaches to investigating calcium/calmodulin-dependent protein kinase II function in plasticity and cognition. J Neurosci 24:8410-8415

El-Husseini A, Schnell E, Dakoji S, Sweeney N, Zhou Q, Prange O, Gauthier-Campbell C, Aguilera-Moreno A, Nicoll RA, Bredt DS (2002) Synaptic strength regulated by palmitate cycling on PSD-95. Cell 108:849-863

Filipowicz W, Bhattacharyya SN, Sonenberg N (2008) Mechanisms of post-transcriptional regulation by microRNAs: are the answers in sight? Nat Rev Genet 9:102-114

Forrest KM, Gavis ER (2003) Live imaging of endogenous RNA reveals a diffusion and entrapment mechanism for nanos mRNA localization in Drosophila. Curr Biol 13:1159-1168

Fujii R, Okabe S, Urushido T, Inoue K, Yoshimura A, Tachibana T, Nishikawa T, Hicks GG, Takumi T (2005) The RNA binding 
protein TLS is translocated to dendritic spines by mGluR5 activation and regulates spine morphology. Curr Biol 15:587-593

Fusco D, Accornero N, Lavoie B, Shenoy SM, Blanchard JM, Singer RH, Bertrand E (2003) Single mRNA molecules demonstrate probabilistic movement in living mammalian cells. Curr Biol 13:161-167

Gao Y, Tatavarty V, Korza G, Levin MK, Carson JH (2008) Multiplexed dendritic targeting of alpha calcium calmodulin-dependent protein kinase II, neurogranin, and activity-regulated cytoskeletonassociated protein RNAs by the A2 pathway. Mol Biol Cell 19:2311-2327

Giorgi C, Yeo GW, Stone ME, Katz DB, Burge C, Turrigiano G, Moore MJ (2007) The EJC factor eIF4AIII modulates synaptic strength and neuronal protein expression. Cell 130:179-191

Goetze B, Tuebing F, Xie Y, Dorostkar MM, Thomas S, Pehl U, Boehm S, Macchi P, Kiebler MA (2006) The brain-specific double-stranded RNA-binding protein Staufen2 is required for dendritic spine morphogenesis. J Cell Biol 172:221-231

Hanz S, Perlson E, Willis D, Zheng JQ, Massarwa R, Huerta JJ, Koltzenburg M, Kohler M, van-Minnen J, Twiss JL, Fainzilber M (2003) Axoplasmic importins enable retrograde injury signaling in lesioned nerve. Neuron 40:1095-1104

Hengst U, Jaffrey SR (2007) Function and translational regulation of mRNA in developing axons. Semin Cell Dev Biol 18:209-215

Hengst U, Cox LJ, Macosko EZ, Jaffrey SR (2006) Functional and selective RNA interference in developing axons and growth cones. J Neurosci 26:5727-5732

Hengst U, Deglincerti A, Kim HJ, Jeon NL, Jaffrey SR (2009) Axonal elongation triggered by stimulus-induced local translation of a polarity complex protein. Nat Cell Biol 11:1024-1030

Hering H, Sheng M (2001) Dendritic spines: structure, dynamics and regulation. Nat Rev Neurosci 2:880-888

Huang YS, Carson JH, Barbarese E, Richter JD (2003) Facilitation of dendritic mRNA transport by CPEB. Genes Dev 17:638-653

Hubel DH, Wiesel TN (1962) Receptive fields, binocular interaction and functional architecture in the cat's visual cortex. J Physiol 160:106-154

Hüttelmaier S, Zenklusen D, Lederer M, Dictenberg J, Lorenz M, Meng X, Bassell GJ, Condeelis J, Singer RH (2005) Spatial regulation of beta-actin translation by Src-dependent phosphorylation of ZBP1. Nature 438:512-515

Jaramillo AM, Weil TT, Goodhouse J, Gavis ER, Schupbach T (2008) The dynamics of fluorescently labeled endogenous gurken mRNA in Drosophila. J Cell Sci 121:887-894

Kanai Y, Dohmae N, Hirokawa N (2004) Kinesin transports RNA: isolation and characterization of an RNA-transporting granule. Neuron 43:513-525

Kiebler MA, Bassell GJ (2006) Neuronal RNA granules: movers and makers. Neuron 51:685-690

Kiebler MA, DesGroseillers L (2000) Molecular insights into mRNA transport and local translation in the mammalian nervous system. Neuron 25:19-28

Köhrmann M, Luo M, Kaether C, DesGroseillers L, Dotti CG, Kiebler MA (1999) Microtubule-dependent recruitment of Staufen-green fluorescent protein into large RNA-containing granules and subsequent dendritic transport in living hippocampal neurons. Mol Biol Cell 10:2945-2953

Kosik KS (2006) The neuronal microRNA system. Nat Rev Neurosci 7:911-920

Lai KO, Zhao Y, Ch'ng TH, Martin KC (2008) Importin-mediated retrograde transport of CREB2 from distal processes to the nucleus in neurons. Proc Natl Acad Sci USA 105:17175-17180

Lange S, Katayama Y, Schmid M, Burkacky O, Brauchle C, Lamb DC, Jansen RP (2008) Simultaneous transport of different localized mRNA species revealed by live-cell imaging. Traffic 9:1256-1267
Lappe-Siefke C, Maas C, Kneussel M (2008) Microinjection into cultured hippocampal neurons: a straightforward approach for controlled cellular delivery of nucleic acids, peptides and antibodies. J Neurosci Methods 175:88-95

Lebeau G, Maher-Laporte M, Topolnik L, Laurent CE, Sossin W, Desgroseillers L, Lacaille JC (2008) Staufen1 regulation of protein synthesis-dependent long-term potentiation and synaptic function in hippocampal pyramidal cells. Mol Cell Biol 28:28962907

Lee R, Kermani P, Teng KK, Hempstead BL (2001) Regulation of cell survival by secreted proneurotrophins. Science 294:1945-1948

Lehmann R, Nüsslein-Volhard C (1991) The maternal gene nanos has a central role in posterior pattern formation of the Drosophila embryo. Development 112:679-691

Leung KM, van Horck FP, Lin AC, Allison R, Standart N, Holt CE (2006) Asymmetrical beta-actin mRNA translation in growth cones mediates attractive turning to netrin-1. Nat Neurosci 9:1247-1256

Link W, Konietzko U, Kauselmann G, Krug M, Schwanke B, Frey U, Kuhl D (1995) Somatodendritic expression of an immediate early gene is regulated by synaptic activity. Proc Natl Acad Sci USA 92:5734-5738

Lyford GL, Yamagata K, Kaufmann WE, Barnes CA, Sanders LK, Copeland NG, Gilbert DJ, Jenkins NA, Lanahan AA, Worley PF (1995) Arc, a growth factor and activity-regulated gene, encodes a novel cytoskeleton-associated protein that is enriched in neuronal dendrites. Neuron 14:433-445

MacDougall N, Clark A, MacDougall E, Davis I (2003) Drosophila gurken (TGFalpha) mRNA localizes as particles that move within the oocyte in two dynein-dependent steps. Dev Cell 4:307-319

Martin KC, Ephrussi A (2009) mRNA localization: gene expression in the spatial dimension. Cell 136:719-730

Meister G, Landthaler M, Peters L, Chen PY, Urlaub H, Luhrmann R, Tuschl T (2005) Identification of novel argonaute-associated proteins. Curr Biol 15:2149-2155

Meng Y, Zhang Y, Tregoubov V, Janus C, Cruz L, Jackson M, Lu WY, MacDonald JF, Wang JY, Falls DL, Jia Z (2002) Abnormal spine morphology and enhanced LTP in LIMK-1 knockout mice. Neuron 35:121-133

Mhlanga MM, Bratu DP, Genovesio A, Rybarska A, Chenouard N, Nehrbass U, Olivo-Marin JC (2009) In vivo colocalisation of oskar mRNA and trans-acting proteins revealed by quantitative imaging of the Drosophila oocyte. PLoS One 4:e6241

Miller S, Yasuda M, Coats JK, Jones Y, Martone ME, Mayford M (2002) Disruption of dendritic translation of CaMKIIalpha impairs stabilization of synaptic plasticity and memory consolidation. Neuron 36:507-519

Nitin N, Santangelo PJ, Kim G, Nie S, Bao G (2004) Peptide-linked molecular beacons for efficient delivery and rapid mRNA detection in living cells. Nucleic Acids Res 32:e58

Oleynikov Y, Singer RH (2003) Real-time visualization of ZBP1 association with beta-actin mRNA during transcription and localization. Curr Biol 13:199-207

Perlson E, Hanz S, Ben-Yaakov K, Segal-Ruder Y, Seger R, Fainzilber $M$ (2005) Vimentin-dependent spatial translocation of an activated MAP kinase in injured nerve. Neuron 45:715-726

Perry RB, Fainzilber M (2009) Nuclear transport factors in neuronal function. Semin Cell Dev Biol 20:600-606

Plath N, Ohana O, Dammermann B, Errington ML, Schmitz D, Gross C, Mao X, Engelsberg A, Mahlke C, Welzl H, Kobalz U, Stawrakakis A, Fernandez E, Waltereit R, Bick-Sander A, Therstappen E, Cooke SF, Blanquet V, Wurst W, Salmen B, Bosl MR, Lipp HP, Grant SG, Bliss TV, Wolfer DP, Kuhl D (2006) Arc/Arg3.1 is essential for the consolidation of synaptic plasticity and memories. Neuron 52:437-444 
Pollard TD, Cooper JA (2009) Actin, a central player in cell shape and movement. Science 326:1208-1212

Rao VR, Pintchovski SA, Chin J, Peebles CL, Mitra S, Finkbeiner S (2006) AMPA receptors regulate transcription of the plasticityrelated immediate-early gene Arc. Nat Neurosci 9:887-895

Rial Verde EM, Lee-Osbourne J, Worley PF, Malinow R, Cline HT (2006) Increased expression of the immediate-early gene arc/ arg3.1 reduces AMPA receptor-mediated synaptic transmission. Neuron 52:461-474

Richter JD, Klann E (2009) Making synaptic plasticity and memory last: mechanisms of translational regulation. Genes Dev 23:1-11

Rook MS, Lu M, Kosik KS (2000) CaMKIIalpha 3' untranslated region-directed mRNA translocation in living neurons: visualization by GFP linkage. J Neurosci 20:6385-6393

Santangelo PJ, Lifland AW, Curt P, Sasaki Y, Bassell GJ, Lindquist ME, Crowe JE Jr (2009) Single molecule-sensitive probes for imaging RNA in live cells. Nat Methods 6:347-349

Schratt GM, Tuebing F, Nigh EA, Kane CG, Sabatini ME, Kiebler M, Greenberg ME (2006) A brain-specific microRNA regulates dendritic spine development. Nature 439:283-289

Shan J, Munro TP, Barbarese E, Carson JH, Smith R (2003) A molecular mechanism for mRNA trafficking in neuronal dendrites. J Neurosci 23:8859-8866

Shepherd JD, Rumbaugh G, Wu J, Chowdhury S, Plath N, Kuhl D, Huganir RL, Worley PF (2006) Arc/Arg3.1 mediates homeostatic synaptic scaling of AMPA receptors. Neuron 52:475-484

Shi SH, Jan LY, Jan YN (2003) Hippocampal neuronal polarity specified by spatially localized mPar3/mPar6 and PI 3-kinase activity. Cell 112:63-75

Siegel G, Obernosterer G, Fiore R, Oehmen M, Bicker S, Christensen M, Khudayberdiev S, Leuschner PF, Busch CJ, Kane C, Hubel K, Dekker F, Hedberg C, Rengarajan B, Drepper C, Waldmann H, Kauppinen S, Greenberg ME, Draguhn A, Rehmsmeier M, Martinez J, Schratt GM (2009) A functional screen implicates microRNA-138-dependent regulation of the depalmitoylation enzyme APT1 in dendritic spine morphogenesis. Nat Cell Biol 11:705-716

Sonenberg N, Hinnebusch AG (2007) New modes of translational control in development, behavior, and disease. Mol Cell 28:721-729

Sonenberg N, Hinnebusch AG (2009) Regulation of translation initiation in eukaryotes: mechanisms and biological targets. Cell 136:731-745

Song HJ, Ming GL, Poo MM (1997) cAMP-induced switching in turning direction of nerve growth cones. Nature 388:275-279

St Johnston D (2005) Moving messages: the intracellular localization of mRNAs. Nat Rev Mol Cell Biol 6:363-375

Steward O, Worley PF (2001) Selective targeting of newly synthesized Arc mRNA to active synapses requires NMDA receptor activation. Neuron 30:227-240

Sutton MA, Schuman EM (2006) Dendritic protein synthesis, synaptic plasticity, and memory. Cell 127:49-58

Taylor AM, Blurton-Jones M, Rhee SW, Cribbs DH, Cotman CW, Jeon NL (2005) A microfluidic culture platform for CNS axonal injury, regeneration and transport. Nat Methods 2:599-605

Thompson KR, Otis KO, Chen DY, Zhao Y, O'Dell TJ, Martin KC (2004) Synapse to nucleus signaling during long-term synaptic plasticity; a role for the classical active nuclear import pathway. Neuron 44:997-1009
Tuebing F, Vendra G, Mikl M, Macchi P, Thomas S, Kiebler M (2010) Dendritically localized transcripts are sorted into distinct RNPs that display fast directional motility along dendrites of hippocampal neurons. J Neurosci (in press)

Tzingounis AV, Nicoll RA (2006) Arc/Arg3.1: linking gene expression to synaptic plasticity and memory. Neuron 52:403-407

Van De Bor V, Hartswood E, Jones C, Finnegan D, Davis I (2005) Gurken and the I factor retrotransposon RNAs share common localization signals and machinery. Dev Cell 9:51-62

Vargas DY, Raj A, Marras SA, Kramer FR, Tyagi S (2005) Mechanism of mRNA transport in the nucleus. Proc Natl Acad Sci USA 102:17008-17013

Vessey JP, Macchi P, Stein JM, Mikl M, Hawker KN, Vogelsang P, Wieczorek K, Vendra G, Riefler J, Tubing F, Aparicio SA, Abel T, Kiebler MA (2008) A loss of function allele for murine Staufen1 leads to impairment of dendritic Staufen1-RNP delivery and dendritic spine morphogenesis. Proc Natl Acad Sci USA 105:16374-16379

Wang KH, Majewska A, Schummers J, Farley B, Hu C, Sur M, Tonegawa $S$ (2006) In vivo two-photon imaging reveals a role of arc in enhancing orientation specificity in visual cortex. Cell 126:389-402

Wang W, van Niekerk E, Willis DE, Twiss JL (2007) RNA transport and localized protein synthesis in neurological disorders and neural repair. Dev Neurobiol 67:1166-1182

Washbourne P, McAllister AK (2002) Techniques for gene transfer into neurons. Curr Opin Neurobiol 12:566-573

Weil TT, Forrest KM, Gavis ER (2006) Localization of bicoid mRNA in late oocytes is maintained by continual active transport. Dev Cell 11:251-262

Weil TT, Parton R, Davis I, Gavis ER (2008) Changes in bicoid mRNA anchoring highlight conserved mechanisms during the oocyteto-embryo transition. Curr Biol 18:1055-1061

Wickham L, Duchaine T, Luo M, Nabi IR, DesGroseillers L (1999) Mammalian staufen is a double-stranded-RNA- and tubulin-binding protein which localizes to the rough endoplasmic reticulum. Mol Cell Biol 19:2220-2230

Wilkie GS, Davis I (2001) Drosophila wingless and pair-rule transcripts localize apically by dynein-mediated transport of RNA particles. Cell 105:209-219

Yao J, Sasaki Y, Wen Z, Bassell GJ, Zheng JQ (2006) An essential role for beta-actin mRNA localization and translation in Ca2+-dependent growth cone guidance. Nat Neurosci 9:1265-1273

Yudin D, Hanz S, Yoo S, Iavnilovitch E, Willis D, Gradus T, Vuppalanchi D, Segal-Ruder Y, Ben-Yaakov K, Hieda M, Yoneda Y, Twiss JL, Fainzilber M (2008) Localized regulation of axonal RanGTPase controls retrograde injury signaling in peripheral nerve. Neuron 59:241-252

Zeitelhofer M, Karra D, Macchi P, Tolino M, Thomas S, Schwarz M, Kiebler M, Dahm R (2008) Dynamic interaction between P-bodies and transport ribonucleoprotein particles in dendrites of mature hippocampal neurons. J Neurosci 28:7555-7562

Zimyanin VL, Belaya K, Pecreaux J, Gilchrist MJ, Clark A, Davis I, St Johnston D (2008) In vivo imaging of oskar mRNA transport reveals the mechanism of posterior localization. Cell 134:843853 